\title{
Imaginarios, creencias y actitudes que influyen en la consulta odontopediátrica en menores de 3 años.
}

\section{Social imaginary, beliefs and attitudes that influence the pediatric dental consultation in children under 3 years old.}

\author{
Valeria Muñoz ${ }^{1 *}$, Patricia M. Valenzuela², Alejandra Rasse ${ }^{3}$.
}

\begin{abstract}
1. Escuela de Odontología. Facultad de Medicina. Pontificia Universidad Católica de Chile. Chile 2. División de Pediatría, Escuela de Medicina, Facultad de Medicina. Pontificia Universidad Católica de Chile. Chile

3. Escuela de Trabajo Social. Pontificia Universidad Católica de Chile. Chile

* Correspondencia Autor: Valeria Muñoz. | Profesor Asistente Adjunto. Escuela de Odontología. Pontificia Universidad Católica de Chile. | Dirección: Av. Vicuña Mackenna 4860, Macul, Santiago, Chile. | Código postal 7820436. | Email: vmunoze@uc.cl Trabajo recibido el 29/09/2019.

Aprobado para su publicación el 17/11/2019
\end{abstract}

\begin{abstract}
RESUMEN
Los niños menores de 3 años son llevados a la consulta médica de forma rutinaria. Sin embargo, la consulta odontológica es muy infrecuente a esta edad. Por otro lado, los elementos que median en la decisión de consultar o no al dentista en esta etapa son hasta el momento poco conocidos. El objetivo de este estudio cualitativo fue identificar imaginarios, creencias y actitudes que podrían intervenir en la decisión de realizar una consulta odontopediátrica en pacientes menores de 3 años. Se aplicaron 10 entrevistas semi-estructuradas a mujeres profesionales, madres de niños entre 8 y 36 meses. Luego, se realizaron 4 entrevistas grupales en profundidad a 13 mujeres que no habían participado en la entrevista inicial. A pesar del alto nivel educativo de las madres se detectó un importante desconocimiento en temas de salud oral. Las madres sub-dimensionan el potencial de la consulta odontopediátrica en esta etapa. En segundo lugar, las madres consideran que una consulta odontopediátrica en este período es abrumadora considerando las altas demandas de esta etapa de la maternidad. Es importante mejorar los conocimientos de salud oral en este grupo de la población y modificar estrategias de prevención y promoción de la salud en preescolares.
\end{abstract}

PALABRAS CLAVE

Relación madre-hijo; Salud oral; Odontopediatría; Pediatría; Investigación Cualitativa; Cuidado Dental.

Int. J. Inter. Dent Vol. 13(1); 06-08, 2020.

\begin{abstract}
Parents of children under 3 years old frequently take their children to medical check-ups. However, dental check-ups are infrequent at this age. The reasons behind this behavior are not well known. The present qualitative study aimed to identify social imaginary, attitudes and beliefs that may influence the decision to consult a pediatric dentist in parents of children under 3 years old. Semi-structured interviews were applied to 10 working mothers that had children between 8 and 36 months. These interviews allowed the identification of the most significant issues to be explored in this study. Afterwards, a total of 13 mothers who did not participate in the initial interviews were divided in 4 groups and in-depth group interviews were performed. Mothers involved in this study had a high educational level. However, their knowledge concerning oral health issues was very poor. Moreover, a frequent finding in this study was the notion that a dental consultation at this stage is considered to be overwhelming by these women because of their very demanding duties. It is important to increase the knowledge regarding oral health topics in this group and to modify policies about oral health at this stage of a child's life.

KEY WORDS

Mother-child relations; Oral health; Pediatric dentistry; Pediatrics; Qualitative research; Dental care.
\end{abstract}

Int. J. Inter. Dent Vol. 13(1); 06-08, 2020.

\section{INTRODUCCIÓN}

Es habitual que los padres lleven a sus hijos a controles médicos durante sus primeros años de vida. Sin embargo, la consulta odontológica es mucho más infrecuente en esta etapa.. De hecho, algunos estudios que arrojan evidencia preliminar sobre este tema y muestran que un bajo porcentaje de los niños de esta edad acude a controles odontológicos preventivos y que los padres consideran la consulta odontológica mayoritariamente frente a situaciones de urgencia ${ }^{(1,2)}$. Podría pensarse que esto responde a que, durante estos años, no ocurre nada relevante en términos de salud bucal.
Sin embargo, en este período existe un importante riesgo de exposición a factores de riesgo que inician las principales enfermedades de salud en la infancia. Existe evidencia que muestra que la gravedad de las caries se correlaciona con el desarrollo infantil temprano en niños de apenas tres años de edad(3). En escolares menores de 6 años, la caries temprana de la infancia afecta el desarrollo, rendimiento académico, bienestar psicosocial y comportamiento de los niños, además de generar estrés en el sistema familiar y en los establecimientos de salud ${ }^{(4,5)}$. Por otro lado, esta etapa aparece como un momento privilegiado para el desarrollo de hábitos saludables. Estos antecedentes hacen pensar que podrían existir elementos 
aún no identificados que median en la decisión de consultar al odontólogo en niños menores de 36 meses y de adoptar hábitos favorables para la prevención de enfermedades de la cavidad oral.

Existen estudios que indican que los padres con mayor conocimiento consultan más y cepillan más los dientes a sus hijos ${ }^{(2,6)}$ y están más dispuestos a invertir tiempo y dinero en la salud oral de sus hijos ${ }^{(7)}$. Esto parece estar mediado por su percepción de autoeficacia(6), y por sus creencias más profundas ${ }^{(8)}$. Sin embargo, los estudios señalan que aún tras recibir información por parte de personal profesional, los padres no cambian sus actitudes respecto a la consulta odontológica en niños pequeños. Esto lleva a pensar que las creencias y experiencias son más fuertes que el conocimiento que se les pueda transmitir.

Para el caso chileno, logró identificar un solo estudio sobre este tema, realizado en 1989 en una muestra de sujetos de bajos recursos, que muestra bajísimos conocimientos sobre salud oral en la población ${ }^{(9)}$. A su vez, en este estudio se detectó la percepción generalizada de que ninguna conducta o hábito que se adopte puede contrarrestar el peso de la genética que sería el factor determinante en el desarrollo de enfermedades ${ }^{(9)}$. Esto es consistente con lo encontrado en estudios más recientes a nivel internacional(10).

En este contexto, el presente estudio realizó una indagación exploratoria en torno a los elementos socioculturales que podrían intervenir en la decisión de consultar al Odontopediatra a temprana edad, respondiendo a la pregunta: ¿Cuáles son los imaginarios, creencias y actitudes en torno a la consulta odontológica de menores de tres años que albergan las madres principales cuidadoras de niños de esta edad? Se espera encontrar en estos imaginarios claves que permitan comprender los motivos que obstaculizan la consulta odontológica en niños y niñas de esta edad.

\section{MATERIAL Y MÉTODO}

La presente investigación corresponde a un estudio cualitativo, de acuerdo al enfoque de la teoría fundamentada. La población se definió como los cuidadores principales de niños entre 6 y 36 meses, profesionales, en ocupaciones profesionales o de servicios no manuales. Si bien la convocatoria fue abierta, en la práctica solo se autoidentificaron como cuidadores principales, las madres. Se definió incluir solo profesionales (técnicos o universitarios) para acceder a los imaginarios de la población que, en principio, podría disponer de mayor información (o al menos de mayor acceso a ella) y menores restricciones económicas.

En una primera etapa, se realizaron 10 entrevistas semi estructuradas a madres de niños en el rango definido, de las cuales la mitad había consultado, y la otra mitad no había llevado nunca a su hijo al odontólogo. Se les preguntó sobre sus imaginarios sobre la consulta odontológica en general, la consulta odontológica pediátrica, hábitos, concepciones y temores en torno a la salud bucal. Este análisis preliminar de estos resultados permitió definir los temas más relevantes a investigar. Luego se diseñó una segunda etapa de profundización, abordando a 13 madres con hijos en la edad definida que se conocían entre ellas y que fueron divididas en 4 grupos. En total se abordó a 23 sujetos. La muestra fue homogénea en términos de género, pero diversa en términos de profesiones, nivel socioeconómico de los hogares, número de hijos, edades de los hijos y las madres, y experiencias en torno a la consulta odontológica.

El conjunto de entrevistadas incorporó criterios de diversidad socioeconómica, de estructura familiar, ocupacional y residencial. Entre las madres entrevistadas hubo mujeres de estrato medio, medio alto y alto. El nivel socio económico se determinó en torno a ocupación y educación de ambos cónyuges, y patrimonio familiar.

Las madres eran profesionales técnicas y universitarias. Las comunas de residencia también fueron variadas. El estudio incluyó madres con uno, dos y tres hijos, así como familias biparentales y monoparentales. Todas las mujeres trabajaban al menos medio tiempo, y en distintos formatos: desde aquellas que trabajaban por proyecto, con total flexibilidad y fluctuación de carga horaria, hasta aquellas que trabajaban jornada completa marcando tarjeta de horario. La tabla 1 muestra las profesiones, comuna de residencia y nivel socioeconómico de las madres entrevistadas.

Todas las entrevistas fueron obtenidas previo proceso de consentimiento informado, el cual fue visado por el Comité de Ética en Investigación de la Pontificia Universidad Católica de Chile. El protocolo de investigación fue el ID16070401. Las entrevistas fueron transcritas de forma literal, y fueron trabajadas con codificación abierta, axial y selectiva. Se logró la saturación de las categorías conceptuales desarrolladas.

\section{RESULTADOS}

\section{Decisión de consulta}

La decisión de consulta está determinada por diversos factores. Un elemento central es la indicación pediátrica. Mientras algunas madres relatan que el pediatra las ha instado a consultar (a los 2 o 3 años), otras
Tabla 1: Profesiones, lugares de residencia y nivel socio económico de las madres entrevistadas.

\begin{tabular}{|c|c|c|}
\hline Ocupación & Comuna & $\begin{array}{c}\text { Nivel } \\
\text { socioeconómico }\end{array}$ \\
\hline Trabajadora Social & Pirque & Medio alto \\
\hline Economista & Las Condes & Alto \\
\hline $\begin{array}{l}\text { Ingeniera en } \\
\text { Alimentos }\end{array}$ & Providencia & Medio alto \\
\hline Asistente & Til Til & Medio \\
\hline Ingeniera Comercial & Ñuñoa & Medio Alto \\
\hline Psicóloga & Macul & Medio \\
\hline Diseñadora Gráfica & Santiago & Medio \\
\hline Trabajadora Social & Maipú & Medio \\
\hline $\begin{array}{l}\text { Ingeniera } \\
\text { Informática }\end{array}$ & Puente Alto & Medio \\
\hline Economista & Santiago & Medio alto \\
\hline Urbanista & Providencia & Medio alto \\
\hline Socióloga & Providencia & Medio alto \\
\hline Veterinaria & Providencia & Medio alto \\
\hline Diseñadora & Providencia & Medio alto \\
\hline Abogada & Providencia & Medio alto \\
\hline Socióloga & La Reina & Alto \\
\hline Socióloga & Ñuñoa & Medio alto \\
\hline Socióloga & Ñuñoa & Medio alto \\
\hline Periodista & Las Condes & Medio alto \\
\hline Arquitecta & Providencia & Alto \\
\hline Actriz & Peñalolén & Medio \\
\hline Profesora de teatro & Ñuñoa & Medio \\
\hline Artista visual & Ñuñoa & Medio \\
\hline
\end{tabular}

señalan que el pediatra nunca les ha referido ninguna orientación en torno al tema odontológico. En estas madres se evidenció en el curso de la entrevista una reflexión respecto a lo importante que es que el pediatra tome un rol más activo en este tema. Para las madres, lo ideal sería que en la consulta pediátrica se dieran instrucciones sobre temas generales en el área de la Odontología tales como edad de inicio del cepillado, uso de pasta dental, entre otros, e información respecto a la edad en que debiera darse la primera consulta odontológica. Salvo casos muy puntuales en que existió orientación previa, las madres no tenían claridad respecto a cuál es la mejor edad para comenzar con la consulta odontológica. Tampoco hubo claridad respecto a la necesidad de esta primera consulta a edad muy temprana. Parte importante de las madres señala que nunca habían pensado en este tema, y que solo cuando se las citó a este estudio pensaron en esto. Otro tema vinculado a la decisión de consulta son las prioridades y tiempos de las madres. Por una parte, la consulta odontológica no es un tema presente para la mayoría de ellas, y pareciera no representar urgencia. Una observación frecuentemente mencionada es que la salud oral cobra importancia a la edad de dos o tres años y que la dentición aparece como un proceso largo y lento. Esto hace que la salud oral pierda importancia respecto a otras interconsultas más urgentes. Por otra parte, las madres consultadas evidencian una enorme sobrecarga de trabajo en su doble rol laboral y doméstico. Coordinar horarios para lograr llevar a sus niños a una consulta se vuelve algo muy complejo, sumado a la dificultad de poder escoger un odontólogo pediátrico que les guste y les dé confianza. La tabla 1 presenta los datos demográficos más relevantes de las pacientes entrevistadas.

\section{Hábitos}

El hábito más frecuentemente señalado como relevante es el cepillado, pero existe una enorme desinformación en torno a este tema, que es reconocida por las madres. Las madres no tienen claridad de cuándo debieran comenzar la práctica del cepillado ni por qué. Los momentos de inicio del cepillado narrados por las madres van desde el inicio de la dentición, en que se utilizan cepillos de silicona pensando principalmente en el alivio más que en la limpieza, hasta el momento en que los niños ya tienen la totalidad de sus dientes y comen todo tipo de alimentos. En algunos casos 
las madres comienzan el cepillado por orientación del pediatra o porque preguntan respecto al tema a algún amigo o familiar. En otros, porque ven en la farmacia que existen productos para cepillar los dientes diseñados a estas edades. También ocurre que la técnica de cepillado se inicia en los niños cuando es indicado en el jardín infantil. En algunas entrevistas, las madres aún no habían comenzado a cepillar los dientes de los niños y señalaron que solo al ser citadas a participar del estudio pensaron en el tema y comenzaron con este hábito. Si bien se otorga importancia al cepillado, las madres señalan que su principal valor es crear el hábito (más que la eficiencia). A medida en que los niños van creciendo y tienen más dientes, las madres se preocupan más por la limpieza. Sin embargo, persiste en algunas de ellas la valoración del cepillado principalmente como un hábito y no como una medida preventiva. La valoración de la técnica de cepillado como un hábito más que por su eficiencia se relaciona con dos elementos. La desvalorización de los dientes primarios o "de leche" y la percepción de que las problemáticas dentales que las madres más temen, como caries y malposiciones dentarias o "dientes chuecos", están determinadas por la genética. Respecto a la importancia de los dientes primarios, hay diversas opiniones, sin embargo, todas coinciden en algo: lo que ocurre con los dientes primarios no es determinante respecto de lo que ocurrirá en el futuro con la salud bucal del niño. Algunas madres señalan que los dientes primarios no tienen ninguna importancia porque se cambiarán. Otras no saben si tendrán alguna importancia, pero piensan que, si la tuvieran, esta sería débil. Sólo en un caso existe conciencia de la posible influencia de los dientes primarios en los dientes definitivos, por la experiencia vivida en el caso de un hijo mayor.

Respecto al uso de chupete y mamadera, las madres consideran que su efecto negativo es un mito, y si bien dan espacio a la duda respecto a su posible capacidad de causar daño en la salud odontológica de los niños, lo relativizan producto de la experiencia ya que ellas mismas $u$ otros usaron chupete o mamadera hasta avanzada edad y perciben que no ocurrió nada.

\section{Imaginarios en torno a la consulta.}

Cuando se pregunta por el ideal de consulta odontopediátrica, todas las madres refieren que debería ser un espacio lúdico, en que se permita el espacio para que el niño se familiarice con el lugar y el profesional, y en lo posible, en que la espera sea corta para que el niño no pierda la paciencia o la buena disposición. Esto se asocia al temor más extendido entre las entrevistadas: el que su hijo sea capaz de estar tranquilo mientras se lo examina. Muchas de estas madres señalan que si su hijo llora o se niega al examen en la consulta pediátrica, lo mismo debería ocurrir en la consulta odontopediátrica. En este marco, consideran que intentar llevar al niño al Odontopediatra puede ser inútil y una pérdida de tiempo en el marco de una agenda difícil, o bien, traumático, dificultando la relación futura con el dentista.

Es importante señalar que hay un grupo de madres, entre las cuales están las que ya han llevado a sus hijos a consultar, que tienen una imagen positiva de la consulta dental. Estas madres corresponden a personas donde ellas mismas tienen una historia de consulta preventiva desde que eran niñas.

\section{Valoración de la consulta}

Entre las madres que ya han llevado a sus hijos a consultar en odontopediatría, en general existe una valoración positiva de la consulta. Se señala que la consulta temprana y periódica puede ayudar a generar en los niños el hábito de ir al dentista, y se valoran las recomendaciones recibidas, en especial las relativas al cepillado y pasta dental.

En relación al cepillado, las madres que asisten a consulta con sus hijos replican las instrucciones para un buen cepillado, pero no necesariamente la periodicidad. Los niños se van a dormir con los dientes cepillados de acuerdo a indicación, pero luego piden leche y duermen el resto de la noche con los dientes sin la debida limpieza. Si bien saben que esto es perjudicial, señalan que es muy difícil erradicar este hábito porque está relacionado con su capacidad para hacer dormir a los niños. En relación a este último punto indican que, (i) no tienen una estrategia clara para cambiar esta costumbre, y (ii) necesitan, en el marco de su nivel de sobredemanda, poder dormir. Resulta interesante que esto también ocurre entre las madres que no han llevado a sus niños a consultar. Respecto de esto último, llama la atención la importancia de las estrategias en el desarrollo y mantención de los hábitos. Aquellas madres que logran una estrategia efectiva mantienen el hábito sin problemas. En la medida que no signifique una batalla, y se incorpore en la rutina. Al contrario, cuando se carece de estrategias alternativas, se postergan las acciones de cambio hasta un momento que parezca más propicio (cuando el niño sea más grande, cuando la mamá esté menos cansada, etc.).

Respecto a las recomendaciones de succión (chupetes y mamaderas) lo recién dicho se refuerza con la relativización de la efectividad de las mismas. Para las madres, parecen no ser tan importantes como la genética. Dado que son recomendaciones difíciles de seguir, se dejan de lado. Las recomendaciones de alimentación tienen mayor adherencia, pero principalmente porque tienen sentido también desde otras dimensiones de la salud.

\section{DISCUSIÓN}

El presente estudio abordó una problemática pobremente evaluada hasta el momento en la población preescolar identificando elementos, hasta el momento desconocidos, que median en la decisión de acudir a una primera consulta al odontólogo. Pese al alto nivel educativo de las madres incluidas en la muestra, la falta de conocimientos sobre prevención y salud oral fue la norma. En este contexto, generalmente subestiman el potencial de la consulta y recomendaciones odontológicas en esta etapa.

En segundo lugar, las condiciones y rutinas cotidianas de este grupo de mujeres las llevan a considerar la consulta odontológica como una sobrecarga, estimando lo sobre demandadas que se sienten en su vida diaria durante este período de la maternidad. Esto se replica respecto de la mantención de los hábitos saludables en sus rutinas diarias. Este hallazgo es consistente con lo descrito en la literatura donde se señala la necesidad de apoyar a los padres proveyéndolos de estrategias que les permitan mantener los hábitos saludables frente a las dificultades cotidianas que experimentan ${ }^{(10)}$. Resulta relevante comprender la influencia de estos elementos en la salud oral, en vistas de modificar las políticas en este tema, la forma de establecer las atenciones y la manera de entregar la información y consejos de salud para pacientes pediátricos y sus cuidadores, sea en servicio público o privado.

Adicionalmente, en esta etapa las recomendaciones del pediatra resultan centrales para la promoción de la salud oral. Lamentablemente, la evidencia disponible señala que la formación y los conocimientos del pediatra en estos temas son más bien débiles, pese a que declaran recibir cotidianamente consultas sobre salud oral(11). En referencia a este tema se ha mencionado que es necesario que los pediatras reciban entrenamiento y formación en salud odontológica, ya que estos, a través de diversas estrategias de prevención e intervención pueden contribuir de manera sustantiva a la salud oral de sus pacientes ${ }^{(12)}$.

\section{FINANCIAMIENTO}

Proyecto financiado con fondos propios.

\section{CONFLICTOS DE INTERÉS}

Autores declaran no tener conflictos de interés.

\section{Bibliografía}

1. Ricci L, Geller D, De Oliveira J, De Andrade M, Fabio A, Coelho M, et al. When and why parents seek dental carefor children under 36 months. Int J Oral Health. 2013;5:21-25.

2. Aljafari A, Gallagher J, Hosey M. Failure on all fronts: General dental practitioners views on promoting oral helth in high caries risk children - a qualitative study. BMC Oral Health. 2015;15:45.

3. Núñez L, Sanz J, Mejía G. Caries dental y desarrollo infantil temprano. Estudio piloto. Rev Chil Pediatr. 2015;86:38-42.

4. Casamassimo P, Thikkurissy S, Edelstein B, Maiorini E. Beyond the dmft. The human and economic costo of early childhood caries. J Am Dent Assoc. 2009;140:650-657.

5. Guarnizo-Herreño C, Wehby G. Children's dental health, school performance, and psychosocial well-being. J Pediatr. 2012;161:1153-1159.

6 . De Silva-Sanigorski, et al. Parental self-efficacy and oral health-related knowledge are associated with parent and child oral health behaviors and self-reported oral health status. Community Dent Oral Epidemiol. 2012;41:345-352.
7. Vermaire J, van Exel N, van Loveren C, Brower W. Putting your money where your mouth is: Parents' valuation of good oral health of their children". Soc Sci Med. 2012;75:2200-2206.

8. Núñez I, Parés G, López R. Evaluación de las actitudes de los padres de familia hacia la primera consulta odontológica de los hijos. Rev Odont Mex. 2006;10:30-35. 9. Misrachi C, Sáez M. Valores, creencias y prácticas populares en relación a la salud oral. Cuad Med Soc. 1989;30:27-33.

10. Duijster D, Jong-Lenters M, Verrips E, Lovern C. Establishing oral health promoting behabiours in children - parents' views on barriers, facilitators and profesional support - a qualitative study" BMC Oral Health 2015;15:157.

11. Lewis $C$, Grossman D, Domoto $P$, Deyo R. The role of pediatrician in the oral health of children: A national survey. Pediatrics. 2000;106:E84

12. Section on Pediatric Dentistry and Oral Health. Preventive oral health intervention for pediatricians". Pediatrics 2008; 122:1387-1394. 